\title{
Effects of pulsed anodic oxide on the intermixing in InGaAs/GaAs and InGaAs/AIGaAs quantum wells
}

\author{
Shu Yuan, ${ }^{\text {a) }}$ C. Y. Liu, and F. Zhao \\ School of Materials Engineering, Nanyang Technological University, Singapore 639798 \\ M. C. Y. Chan and W. K. Tsui \\ Department of Electrical and Electronic Engineering, The University of Hong Kong, Hong Kong \\ L. V. Dao \\ CAOUS, Swinburne University of Technology, Melbourne, VIC-3122, Australia \\ X. Q. Liu \\ Department of Electronic Materials Engineering, Australian National University, Canberra, \\ ACT 0200, Australia
}

(Received 19 December 2002; accepted 2 April 2003)

\begin{abstract}
Intermixing in InGaAs/GaAs and InGaAs/AlGaAs quantum well structures after rapid thermal annealing with and without an anodic oxide cap on the surface was studied by low temperature ( 8 K) photoluminescence (PL). The PL peak energy was shifted towards higher photon energies (blueshift) in both types of samples, especially at annealing temperatures above $880^{\circ} \mathrm{C}$. The anodic oxide cap has been demonstrated to inhibit the band-gap blueshift of the quantum well structures. Secondary ion mass spectroscopy data indicated that Ga vacancies were injected from the anodic oxide cap into the epitaxial layers. These vacancies enhanced interdiffusion between group III atoms, and partially relaxed the strain in the structure, resulting in the effect of the suppression of the blueshift. (C) 2003 American Institute of Physics. [DOI: 10.1063/1.1577407]
\end{abstract}

\section{INTRODUCTION}

Intermixing in semiconductor quantum well structures is of interest due to its applications to semiconductor lasers, transparent waveguides, and optoelectronic integrated circuits. ${ }^{1}$ Intermixing between quantum wells (QWs) and adjacent barriers is usually achieved by interdiffusion between constituent atoms during high temperature annealing, and results in a change in the QW shape, which in turn modifies the subband energy in the conduction and the valence bands. ${ }^{2}$ As the refractive index is directly related to absorption coefficient, ${ }^{3}$ intermixing also modifies the refractive index. There are currently two kinds of intermixing techniques, one is impurity-induced intermixing (IID), ${ }^{4,5}$ the other is impurity-free intermixing (IFID) ${ }^{6-8}$ IID usually introduces substantial undesired changes in the material resistivity and trap concentrations. IFID can create large band-gap energy shifts without such problems associated with IID. $\mathrm{SiO}_{2}$ and $\mathrm{Si}_{3} \mathrm{~N}_{4}$ are commonly used to promote and prevent intermixing, respectively. Laser induced intermixing is also used in the InGaAsP system. ${ }^{9}$

We have previously demonstrated that anodic oxide enhances intermixing in the GaAs/AlGaAs quantum well system. $^{10,11}$ We proposed that the anodic oxide absorbs Ga atoms from the epitaxial layers beneath, and thus injects $\mathrm{Ga}$ vacancies into the quantum well. The Ga vacancies promote interdiffusion of group-III atoms, i.e., $\mathrm{Ga}$ and $\mathrm{Al}$ atoms. The enhanced intermixing was applied to increase photolumines-

a) Author to whom correspondence should be addressed; electronic mail: assyuan@ntu.edu.sg cence (PL) and cathodoluminescence efficiencies significantly from V-grooved GaAs/AlGaAs quantum wires. ${ }^{12,13}$ The effect of the anodic oxide on the quantum well intermixing (QWI) in the InGaAs/AlGaAs (including InGaAs/GaAs) system has not been studied so far. The QWI in the InGaAs/ AlGaAs system has been less studied compared to the GaAs/ AlGaAs system. ${ }^{14-18}$ This system is characterized by the strain in the quantum well. The strain effects on the QWI are controversial. Ryu et al. ${ }^{14}$ studied the effects of strain on the interdiffusion in InGaAs/GaAs quantum wells, and concluded that strain influenced the diffusivity. Li et al. ${ }^{16}$ stated that strain effects appear to enhance disordering during the early stage of the anneal. Gillin, ${ }^{15}$ however, reported no effect of strain on the diffusion in the InGaAs/GaAs system.

In this work, we report the effects of pulsed anodic oxide on the intermixing in InGaAs/AlGaAs quantum wells. Samples with and without anodic oxide were annealed by rapid thermal annealing (RTA) and characterized by $8 \mathrm{~K}$ PL measurements. Noticeable blueshifts of the PL peak energy in both types of samples were observed after annealing at temperatures higher than $880^{\circ} \mathrm{C}$, but the blueshift was suppressed in the samples with anodic oxide cap layers. We propose that the anodic oxide injects $\mathrm{Ga}$ vacancies into the semiconductors. The Ga vacancies' effects on the interband transition energy (and thus the PL peak energy) are twofolded: (1) to enhance the interdiffusion between group III atoms (In and $\mathrm{Ga}$ or $\mathrm{Al}$ ), which leads to an enhanced blueshift as in the GaAs/AlGaAs system (2) to partially relax the strain in the semiconductor, which results in a lower interband transition energy. The net effect was a suppression of the blueshift as manifested from the PL measurements. This 
TABLE I. Sample structures. All epilayers are undoped and their thicknesses are nominal.

\begin{tabular}{llll}
\hline \hline Sample A & \multicolumn{3}{c}{ Sample B } \\
\hline GaAs & $150 \mathrm{~nm}$ & $\mathrm{GaAs}$ & $400 \mathrm{~nm}$ \\
$\mathrm{In}_{0.19} \mathrm{Ga}_{0.81} \mathrm{As}(\mathrm{QW} 4)$ & $2 \mathrm{~nm}$ & $\mathrm{Al}_{0.3} \mathrm{Ga}_{0.7} \mathrm{As}$ & $20 \mathrm{~nm}$ \\
$\mathrm{GaAs}$ & $50 \mathrm{~nm}$ & $\mathrm{In}_{0.3} \mathrm{Ga}_{0.7} \mathrm{As}$ & $6 \mathrm{~nm}$ \\
$\mathrm{In}_{0.19} \mathrm{Ga}_{0.81} \mathrm{As}(\mathrm{QW} 3)$ & $4 \mathrm{~nm}$ & $\mathrm{Al}_{0.3} \mathrm{Ga}_{0.7} \mathrm{As}$ & $3000 \mathrm{~nm}$ \\
$\mathrm{GaAs}$ & $50 \mathrm{~nm}$ & $\mathrm{GaAs}$ & $200 \mathrm{~nm}$ \\
$\mathrm{In}_{0.19} \mathrm{Ga}_{0.81} \mathrm{As}(\mathrm{QW} 2)$ & $6 \mathrm{~nm}$ & $p^{+}-\mathrm{GaAs}$ substrate \\
$\mathrm{GaAs}$ & $50 \mathrm{~nm}$ & & \\
$\mathrm{In}_{0.19} \mathrm{Ga}_{0.81} \mathrm{As}(\mathrm{QW} 1)$ & $10 \mathrm{~nm}$ & & \\
$\mathrm{GaAs}^{1000 \mathrm{~nm}}$ & \\
$p^{+}$-GaAs substrate & & & \\
\hline \hline
\end{tabular}

article is arranged as follows: In Sec. II, the sample structures and experimental details are described; in Sec. III, the modeling of the experimental results is outlined; and in Sec. IV the results are presented and discussed. We conclude in Sec. V.

\section{EXPERIMENTAL DETAILS}

Two structures (samples A and B) were grown at $750{ }^{\circ} \mathrm{C}$ by low pressure metal organic chemical vapor deposition (MOCVD) on (001) p-GaAs substrates, which were $\mathrm{Zn}$ doped to a carrier concentration of $\sim 1 \times 10^{19} \mathrm{~cm}^{-3}$ and $2^{\circ}$ off $\langle 100\rangle$ towards $\langle 110\rangle$. Sample A was a multiple quantum well (MQW) structure, while sample B was a single quantum well (SQW) structure. The layer structures for these samples are listed in Table I. All epilayers were nominally undoped. The growth details were reported in Ref. 19.

After the growth, the wafers were cleaved into $8 \mathrm{~mm}$ $\times 8 \mathrm{~mm}$ pieces, similar to what has been described elsewhere. ${ }^{11}$ Half of each piece was then covered with thermal glue. Each sample was rinsed in $10 \% \mathrm{HCl}$ and then deionized water, and nitrogen blown dried. The pulsed anodization was carried out at room temperature, the details were given in Refs. 11 and 20. For sample A, the anodization current density was $120 \mathrm{~mA} / \mathrm{cm}^{2}$, the pulse width was $1 \mathrm{~ms}$ and the period was $12 \mathrm{~ms}$, and the voltage applied was $50 \mathrm{~V}$. For sample B, the anodization current density was 150 $\mathrm{mA} / \mathrm{cm}^{2}$, the pulse width was $0.7 \mathrm{~ms}$, the period was $20 \mathrm{~ms}$, and the voltage applied was $150 \mathrm{~V}$. For both samples, the anodization time was $4 \mathrm{~min}$. The area covered with thermal glue was unanodized, while anodization took place in the uncovered area to form an oxide layer. The parameters were chosen in order to form uniform oxides. The resulting anodic oxide thickness was determined by surface profiling to be $(110 \pm 3)$ and $(140 \pm 2) \mathrm{nm}$ for samples A and B, respectively. After the anodization, the samples were rinsed with deionized water, followed by acetone to remove the thermal glue, then by deionized water and nitrogen blown dried. Each sample was then cleaved into two $4 \mathrm{~mm} \times 8 \mathrm{~mm}$ pieces, so that each piece had half of the area anodized $(4 \mathrm{~mm}$ $\times 4 \mathrm{~mm})$ and half of the area unanodized $(4 \mathrm{~mm} \times 4 \mathrm{~mm})$.

The intermixing was carried out in a rapid thermal annealing chamber with GaAs proximity caps in flowing argon ambient (see the inset of Fig. 5). The samples were placed in the center area of a 6 in. Si wafer, and the temperature was maintained to be within $\pm 5^{\circ} \mathrm{C}$ across the whole 6 in. wafer. The temperature ramp rate was $50^{\circ} \mathrm{C} / \mathrm{s}$. The chamber had been purged with Ar gas at $51 / \mathrm{min}$ for 5 min before the temperature was ramped up and then maintained at $21 / \mathrm{min}$ for $20 \mathrm{~min}$.

PL measurements were performed at $8 \mathrm{~K}$ using an Ar ion laser beam (the $514.5 \mathrm{~nm}$ line), a spectrometer, and a $\mathrm{Si}$ detector connected to a lock-in amplifier. Each time, a few pieces were mounted together on the cold finger of the cryostat and measured one by one.

\section{MODELING OF THE EXPERIMENTAL DATA}

In this work, the QWI was experimentally characterized by photoluminescence measurements. The blueshift (i.e., shift towards higher photon energies) in the PL peak energy is a result of the changes in the band structure after the QWI. Due to In and $\mathrm{Ga}$ (or $\mathrm{Al}$ ) interdiffusion, the quantum well shape changes from square to a smoothed one, which is usually described by the error function. This results in a change in the electron and hole subband energies, and thus the change (blueshift) in the PL energy. The QWI is described by the diffusion coefficient and activation energy. Therefore, a calculation of the interband transition energy, which takes into account the strain change in the quantum well and changes in carrier confinement profiles due to Ga, In, and/or $\mathrm{Al}$ interdiffusion at RTA temperatures, will determine the diffusion coefficient and shed light on the complex interdiffusion processes.

We first calculated the subband energies and wave functions in an intermixing modified single nonsquare quantum well, ${ }^{2}$ and then compared the calculated interband transition energies to the PL data. The confinement profile of this interdiffused quantum well was nonlinear and was modeled here by the error function. The strain effects were also taken into account. The details of the model without strain effects were given in our previous work on anodic-oxide-induced intermixing in GaAs/AlGaAs quantum wells, ${ }^{11}$ where the calculated $\mathrm{Al}$ profile after intermixing was in good agreement with the measured data.

The extent of the interdiffusion process is generally characterized by a diffusion length $L_{d}$, defined here as $L_{d}$ $=\sqrt{D t},{ }^{21}$ where $D$ and $t$ are the In-Ga (or Al) interdiffusion coefficient and annealing time, respectively. We assumed an isotropic interdiffusion of In and $\mathrm{Ga}$ (or $\mathrm{Al}$ ) with the diffusion coefficients being independent of their respective concentrations, which were also assumed to be equal and constant, i.e., we assumed Fick's second law of diffusion applies. $^{22}$ The diffused In composition profile, $w(z)$, across the QW structure is given by ${ }^{22}$

$$
w(z)=\frac{w_{o}}{2}\left[\operatorname{erf}\left(\frac{L_{z}+2 z}{4 L_{d}}\right)+\operatorname{erf}\left(\frac{L_{z}-2 z}{4 L_{d}}\right)\right],
$$


where $w_{o}$ is the as-grown In mole fraction in the well, $L_{z}$ is the as-grown width of the $\mathrm{QW}$, and $z$ is the coordinate along the growth direction (QW centered at $z=0)$ ). erf denotes the error function. ${ }^{23}$

Lattice mismatch between the thin well and the barrier QW materials can be taken up by the in-plane strain. The biaxial in-plane strain and uniaxial shear strain after interdiffusion are given $b^{24,25}$

$$
\begin{aligned}
& \varepsilon_{x x}=\varepsilon_{y y}=\varepsilon(w), \\
& \varepsilon_{z z}=-2\left[c_{12}(w) / c_{11}(w)\right] \varepsilon(w), \\
& \varepsilon_{x y}=\varepsilon_{x z}=\varepsilon_{z y}=0,
\end{aligned}
$$

where $\varepsilon(w)$ is the misfit factor between the well and the barrier, which is negative for compressive strain. The parameters $c_{11}(w)$ and $c_{12}(w)$ are the elastic stiffness constants. The change in the bulk band gap, $S_{\perp}(w)$, as a result of the hydrostatic strain, is given by

$$
S_{\perp}(w)=-2 a(w)\left[1-c_{12}(w) / c_{11}(w)\right] \varepsilon(w) .
$$

The hydrostatic deformation potential $a(w)$ is calculated from

$$
a(w)=-\frac{1}{3}\left[c_{11}(w)+2 c_{12}(w)\right] \frac{d E_{g}}{d P},
$$

where $d E_{g} / d P$ is the hydrostatic pressure coefficient of the lowest direct energy gap $E_{g}$. The splitting energy, $S_{\|}(w)$, between the heavy-hole $(\mathrm{HH})$ and light-hole $(\mathrm{LH})$ band edges induced by the shear component of the strain is

$$
S_{\|}(w)=-b(w)\left[1+2 c_{12}(w) / c_{11}(w)\right] \varepsilon(w),
$$

where $b(w)$ is the shear deformation potential. The coupling between the LH and split-off band results in asymmetric heavy-hole to light-hole splitting. We have

$$
\begin{aligned}
S_{\| \mathrm{HH}}(w)= & S_{\|}(w), \\
S_{\| \mathrm{LH}}(w)= & -\frac{1}{2}\left[S_{\|}(w)+\Delta_{0}(w)\right] \\
& +\frac{1}{2}\left[9 S_{\|}(w)+\Delta_{0}(w)^{2}-2 S_{\|}(w) \Delta_{0}(w)\right]^{1 / 2},
\end{aligned}
$$

where $\Delta_{0}(w)$ is the spin-orbit splitting. The QW confinement potential, $U_{r}(w)$, after the intermixing process is obtained by modifying the unstrained potential profile, $\Delta E_{r}(w)$, which is defined as $\Delta E_{r}(w)=Q_{r}\left[E_{g}(w)-E_{g}(z=0)\right]$, where $E_{g}(w)$ is the interdiffused band gap, i.e., $E_{g}(w)$ $=E_{g}[w(z)], Q_{r}$ is the band offset splitting, and the subscript $r$ denotes either the electron in the conduction band $(C)$, heavy or light holes in the valence band $(V=H$ or $L)$. The QW confinement potential is given by

$$
U_{r}(w)=\Delta E_{r}(w)-S_{\perp r}(w) \pm S_{\| r}(w),
$$

where $S_{\perp r}(w)=Q_{r} S_{\perp}(w)$. The "+" and "-" signs represent the confined $\mathrm{HH}$ and $\mathrm{LH}$ profiles, respectively, and $S_{\| c}(w)=0$.

The diffused QW subband edge $\left(k_{\|}=0\right)$ at the zone center of the $\Gamma_{6}$-valley symmetry can be calculated separately for the electron and holes, using the Ben-Daniel and Duke model $^{26}$ with a $z$-position dependent effective mass on the

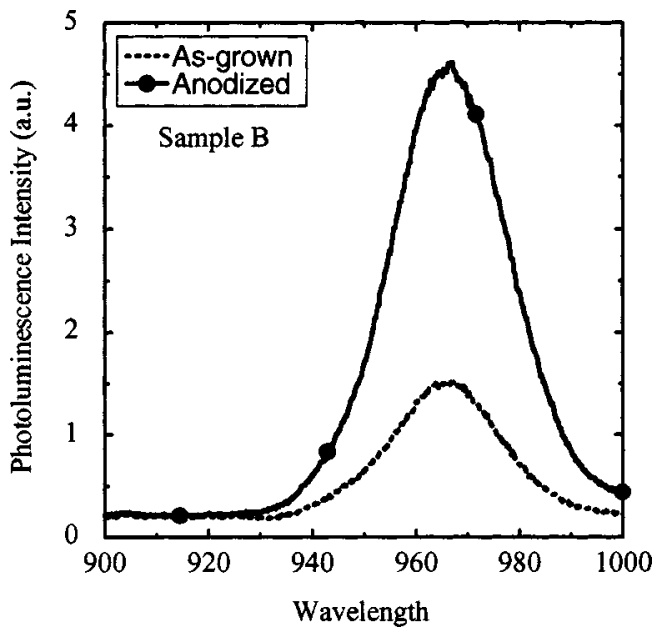

FIG. 1. Comparison of $8 \mathrm{~K}$ PL spectra between as-grown and anodized samples (sample B, InGaAs/AlGaAs SQW). No RTA was carried out on these samples.

interdiffused composition profile, by the one-dimensional Schrödinger-like equation, for the envelope function $\psi_{r l}(z)$, which is written as follows:

$$
-\frac{h^{2}}{2} \frac{d}{d z}\left[\frac{1}{m_{\perp r}^{*}(z)} \frac{d \Psi_{r l}(z)}{d z}\right]+U_{r}(z) \Psi_{r l}(z)=E_{r l} \Psi_{r l}(z),
$$

where $l=1,2, \ldots$, are the QW subband levels for either the electrons $(C l)$ or holes $(V l)$, respectively; $m_{\perp_{r}}^{*}[w(z)]$ is the carrier effective mass in the $z$ direction; $E_{r l} \equiv E_{r l}\left(k_{\|}=0\right)$ is the subband-edge energy, and the origin of the potential energy is taken at the bottom of the diffused QW. Equation (9) was solved numerically using a finite difference method with the corresponding confinement profile with the boundary condition taken to be zero at the end of a finitely high (energy) and thick ( $\geqslant 50 \mathrm{~nm}$ ) barrier.

The calculated quantum well interband transition energies were fitted to the measured PL peak energies by adjusting the value of $D$, thus $D$ can be determined.

\section{RESULTS AND DISCUSSION}

\section{A. Suppression of the blueshift by anodic oxide}

Before the rapid thermal annealing, we first tested the effect of anodic oxide on the band structure of the samples by measuring the photoluminescence on the anodized and unanodized areas of the same sample. As shown in Fig. 1 for sample B (InGaAs/AlGaAs SQW), no noticeable difference in PL peak energy, efficiency, and linewidth was observed, indicating no stress was introduced by the anodic oxide (at least at the PL temperature, i.e., $8 \mathrm{~K}$ ). The PL intensity, however, was noticeably increased by the anodic oxidation. This was likely due to the surface passivation by the anodic oxidation, which reduced the number of surface states. In addition, the oxidation process also consumed some GaAs material in the cap layer $(\sim 110-150 \mathrm{~nm})$, thus more photoelectrons diffused into the quantum well during the PL measurements, resulting in higher PL intensity. 


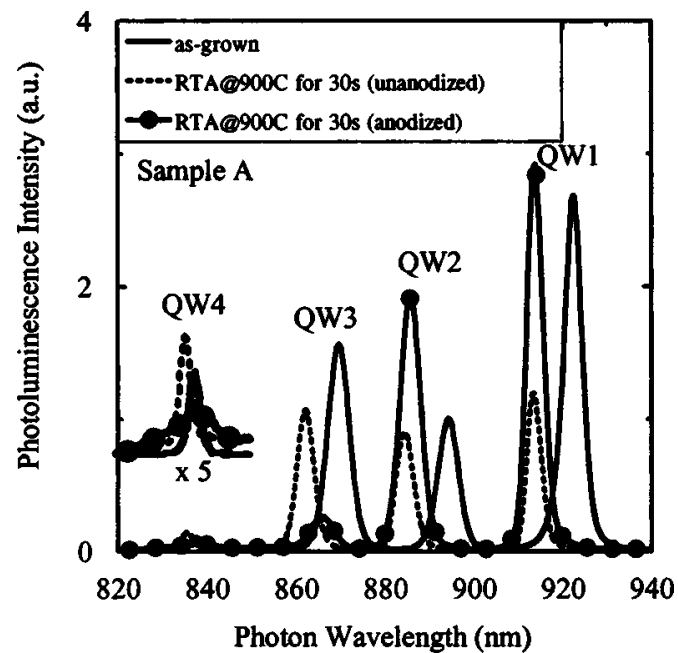

FIG. 2. $8 \mathrm{~K}$ PL spectra from sample A (InGaAs/GaAs MQW). (a) as grown (solid line), (b) annealed without anodic oxide (dashed line), and (c) annealed with anodic oxide (solid dotted line). For (b) and (c), the samples were annealed together at $900{ }^{\circ} \mathrm{C}$ for $30 \mathrm{~s}$.

Figure 2 compares the $8 \mathrm{~K}$ photoluminescence spectra of three samples: (1) as grown, (2) unanodized but annealed, and (3) anodized and annealed samples (sample A, InGaAs/ GaAs MQW). The unanodized and anodized areas of sample A were annealed together in the same run (see the inset in Fig. 5) at $900{ }^{\circ} \mathrm{C}$ for $30 \mathrm{~s}$.

Regarding the as-grown sample A, four peaks were seen, each corresponding to one of the four quantum wells. The assignment of the four quantum wells was based on the calculated interband transition energies using the model outlined in Sec. III with strain effects included in the calculation. QW4 was a shallow well (nominal thickness: $2 \mathrm{~nm}$ ), the electron confinement was quite well, so the PL signal was also weak.

Regarding the unanodized but annealed sample A, after annealing at $900{ }^{\circ} \mathrm{C}$ for $30 \mathrm{~s}$, the PL peaks moved to shorter wavelengths as a consequence of the interdiffusion between In atoms in the quantum well layers and $\mathrm{Ga}$ atoms in the barrier layers. The interdiffusion changed the confinement profiles from square ones to smooth ones, increasing the separation between electron and hole sublevels in the quantum well (i.e., the interband transition energy). ${ }^{11}$ On the other hand, since the interdiffusion of In and $\mathrm{Ga}$ atoms reduced the lattice difference between the well and the barrier layers, it also reduced the compressive strain in the quantum well and tensile strain in the barrier layers. Since the compressive strain in the quantum well increases the energy separation between conduction band electrons and valence band heavy holes, the reduction in the strain by interdiffusion thus reduced the interband transition energy. The net effect of interdiffusion on the interband transition energy in the unanodized sample was the blueshift of PL energy seen in Fig. 1. The blueshift has been reported in the InGaAs/GaAs quantum well systems after QWI. ${ }^{14-17}$

Regarding the anodized and annealed sample A, after annealing with anodic oxide, however, the PL peak energy showed less blueshift compared to those annealed without anodic oxide, indicating the anodic oxide played a role dur-

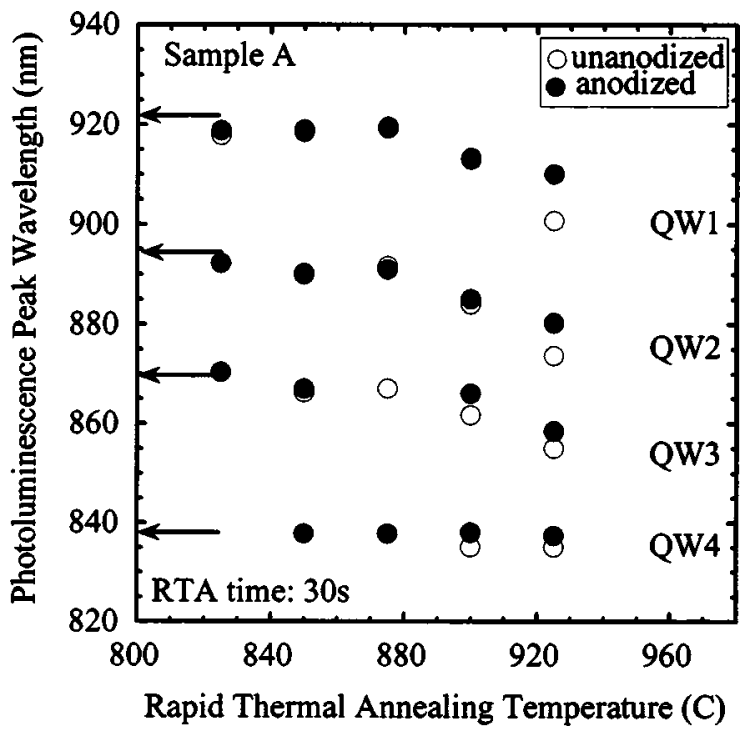

FIG. 3. PL peak wavelength as a function of RTA temperature for QW1-4 in sample A (InGaAs/GaAs MQW), with (filled circles) and without anodic oxide (open circles). The RTA time was $30 \mathrm{~s}$, and PL was taken at $8 \mathrm{~K}$. Arrows indicate the PL position for the as-grown sample.

ing the annealing. The reduced blueshift could be a consequence of two possible reasons: (1) a suppressed interdiffusion between In and $\mathrm{Ga}$ atoms by the anodic oxide during RTA or (2) a partially relax in strain in the semiconductor layers. The mechanism behind this suppression of blueshift will be discussed in latter parts of this article.

Figure 3 shows the PL peak wavelength versus RTA temperature for a fixed annealing time of $30 \mathrm{~s}$ for sample A (InGaAs/GaAs system). The sample was relatively thermally stable up to about $880^{\circ} \mathrm{C}$, then started to show a blueshift. The blueshift was stronger for thicker quantum wells, as there was more room in thicker quantum wells for the electron and hole sublevels to be pushed up by the interdiffusion. This is in agreement with the QWI in GaAs/AlGaAs multiple quantum wells. ${ }^{10}$ For QW1, the unanodized sample showed a blueshift of $22 \mathrm{~nm}$ (33 meV), while the anodized sample was only blueshifted by $12 \mathrm{~nm}(17 \mathrm{meV})$, yielding a reduction in the blueshift by $16 \mathrm{meV}$. It is clear that anodic oxide reduced the blueshift during RTA for all the quantum wells at temperatures above about $880^{\circ} \mathrm{C}$.

In Fig. 4, the PL peak wavelength versus RTA time is shown for $925^{\circ} \mathrm{C}$ anneals as a function of RTA time. When the annealing time was short (1-5 s), instead of a blueshift, a redshift was observed for QW4 (both anodized and unanodized samples) and for QW3 (anodized samples only). QW4 and QW3 were closer to the top surface than QW2 and QW1. This might be understood by the onset of partial strain relieving during the initial stage of the RTA, when interdiffusion between In and $\mathrm{Ga}$ atoms was not significant. The mechanism of strain relieving is the thermally induced dislocation and defect movement to remove partially internal strains. $^{27}$ This partial strain relieving caused a reduction in the energy separation of electrons and holes in the strained InGaAs quantum well. At this early stage of the RTA, the temperature was higher on the surface of the sample than the interior, thus the local temperatures of QW4 and QW3 were 


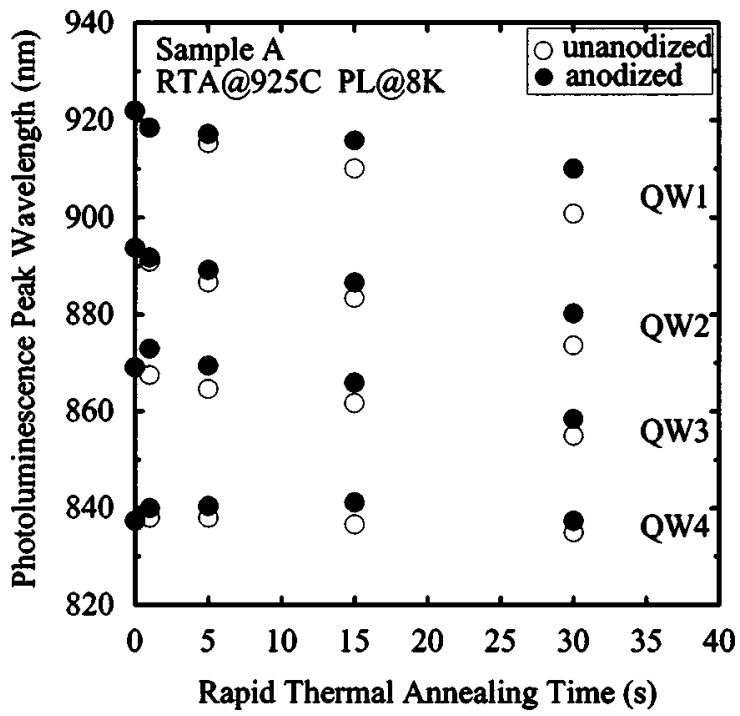

FIG. 4. PL peak wavelength as a function of RTA time for QW1-4 in sample A (InGaAs/GaAs MQW), with (filled circles) and without (open circles) anodic oxide. The RTA temperature was $925^{\circ} \mathrm{C}$ and the PL was taken at 8 $\mathrm{K}$.

higher than QW2 and QW1, the slight redshift in the PL due to the onset of the partial thermal strain relieving was only observed in QW4 and QW3. For longer RTA durations, it reverted to a blueshift due to the interdiffusion. Again, the anodic oxide reduced the blueshift in this $\mathrm{InGaAs} / \mathrm{GaAs}$ quantum well structure for all the four quantum wells.

While sample A belonged to the InGaAs/GaAs system, sample B belonged to the InGaAs/AlGaAs system, and had only one quantum well. Figure 5 shows the PL peak wavelength as a function of RTA time for sample B annealed at $900{ }^{\circ} \mathrm{C}$. After annealing at $900^{\circ} \mathrm{C}$ for $90 \mathrm{~s}$, the unanodized area had a strong blueshift of $41 \mathrm{~nm}(57 \mathrm{meV})$, while the anodized sample had a blueshift of $24 \mathrm{~nm}(33 \mathrm{meV})$, corre-

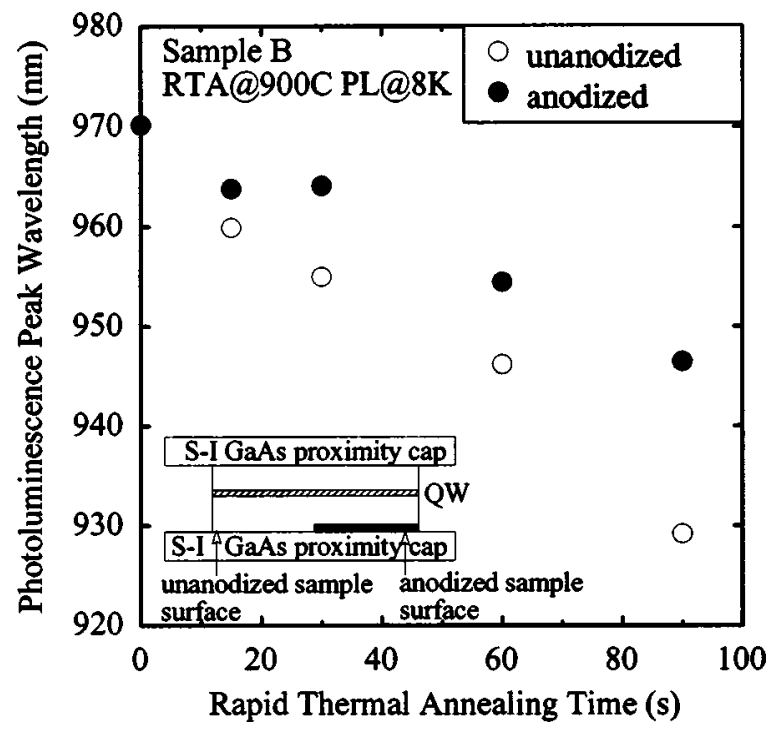

FIG. 5. PL peak wavelength as a function of RTA time sample B (InGaAs/ $\mathrm{AlGaAs} \mathrm{SQW}$ ), with (filled circles) and without (open circles) anodic oxide. The RTA temperature was $900{ }^{\circ} \mathrm{C}$ and the PL was taken at $8 \mathrm{~K}$. The inset shows how a sample was placed in the RTA chamber.

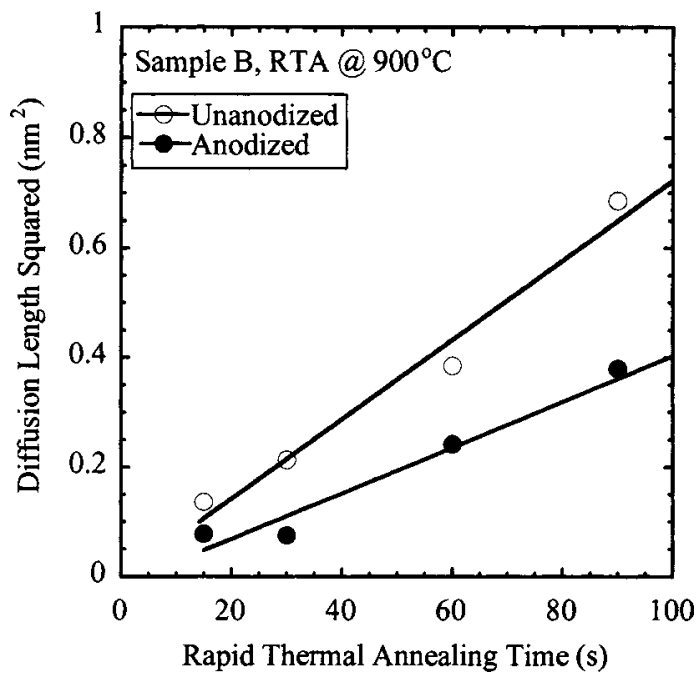

FIG. 6. Diffusion length squared vs annealing time for sample B (InGaAs/ GaAs SQW) annealed at $900{ }^{\circ} \mathrm{C}$ with (filled circles) and without (open circles) anodic oxide. The straight lines are the least-square fit to the data. The fitting resulted in diffusion coefficients $D$ of $(7.3 \pm 0.9) \times 10^{-17}$ and $(4.1 \pm 0.7) \times 10^{-17} \mathrm{~cm}^{2} / \mathrm{s}$ for unanodized and anodized samples, respectively.

sponding to a reduction in the blueshift by $24 \mathrm{meV}$ by the anodic oxide. Clearly, anodic oxide reduced the blueshift in the InGaAs/AlGaAs quantum well structure as well.

\section{B. Diffusion coefficients}

To simply extract the diffusion coefficients for anodized and unanodized samples, we used the method outlined in Sec. III, and treated anodized and unanodized samples the same way, except for having different diffusion coefficients. That is, we used only one parameter (diffusion coefficient) to include the effects of anodic oxide on the band structures in the calculation, although this was certainly an oversimplified approach. Although a more proper method should include the effects of Ga vacancy injection due to anodic oxide on the strain and the diffusivity, the effects are not clearly understood yet, and thus difficult to incorporate in the calculation. Figure 6 is a plot of diffusion length squared versus anneal time for sample B annealed at $900^{\circ} \mathrm{C}$ with and without the anodic oxide cap. The data are more or less linear with anneal time, implying that the diffusion coefficient was constant during the RTA. A least-square fitting resulted in diffusion coefficients $D$ of $(7.3 \pm 0.9) \times 10^{-17}$ and $(4.1 \pm 0.7)$ $\times 10^{-17} \mathrm{~cm}^{2} / \mathrm{s}$ for the unanodized and anodized samples, respectively. Gillin obtained a diffusion coefficient of $\sim 8$ $\times 10^{-17} \mathrm{~cm}^{2} / \mathrm{s}$ for InGaAs/GaAs quantum wells lying at 250 $\mathrm{nm}$ beneath the surface at $900{ }^{\circ} \mathrm{C},{ }^{15}$ which is comparable to our result of $7.3 \times 10^{-17} \mathrm{~cm}^{2} / \mathrm{s}$ for unanodized sample B at $900{ }^{\circ} \mathrm{C}$.

\section{Mechanism of the suppression of blueshift by anodic oxide}

From previous work on the GaAs/AlGaAs system, we have shown that anodic oxide enhances the intermixing in GaAs/AlGaAs quantum wells as manifested from the blueshift in PL measurements. ${ }^{13}$ From the experimental data re- 
TABLE II. Measured (PL) and calculated $\left(E_{11}\right)$ interband transition energies for samples A (QW1) and B (single QW). The last column shows the strain effect, i.e., the difference between the calculated interband transition energies with and without strain.

\begin{tabular}{lcccc}
\hline \hline Sample & $\begin{array}{c}\text { PL }(\mathrm{eV}) \\
\text { (as grown) }\end{array}$ & $\begin{array}{c}E_{11}(\mathrm{eV}) \\
\text { (with strain) }\end{array}$ & $\begin{array}{c}E_{11}(\mathrm{eV}) \\
\text { (without strain) }\end{array}$ & $\begin{array}{c}\text { Strain effect } \\
(\mathrm{meV})\end{array}$ \\
\hline $\mathrm{A}(\mathrm{QW} 1)$ & 1.345 & 1.344 & 1.278 & 66 \\
$\mathrm{~B}(\mathrm{SQW})$ & 1.278 & 1.273 & 1.177 & 96 \\
\hline \hline
\end{tabular}

ported here, however, it is clear that anodic oxide suppresses the blueshift in InGaAs/AlGaAs (including InGaAs/GaAs) quantum wells. Other dielectric caps have been shown to suppress intermixing for various reasons. For example, the suppression of intermixing by a phosphorus doped $\mathrm{SiO}_{2}$ cap was attributed to the fact that $\mathrm{SiO}_{2}: \mathrm{P}$ is more dense and void free compared with standard $\mathrm{SiO}_{2}$ together with a strain relaxation effect of the cap layer during annealing. ${ }^{28}$

It should be pointed out that the suppression of the blueshift in our work does not necessarily mean a suppression of the QWI. In the GaAs/AlGaAs system, the enhanced intermixing by the anodic oxide at RTA temperatures was attributed to the injection of Ga vacancies into the quantum well. ${ }^{13}$ Since the top layer of both our GaAs/AlGaAs and InGaAs/ $\mathrm{AlGaAs}$ samples was GaAs and the barrier layers were Al$\mathrm{GaAs}$, and the anodic oxidation was carried out under similar conditions, it is likely that the QWI in the InGaAs/(Al)GaAs system was still enhanced by the anodic oxide cap during RTA through the injection of Ga vacancies, but a possible strain reduction by the anodic oxide cap caused the reduction in the blueshift. We will discuss this in the following.

The major differences between GaAs/AlGaAs and InGaAs/AlGaAs quantum wells are that strain and indium are present in the InGaAs/AlGaAs system only. Strain was reported to enhance the intermixing in InGaAs/GaAs system, ${ }^{16,17}$ and was thought to play a role during the early stage of rapid thermal annealing. ${ }^{17}$ Gillin, however, observed no effect of strain on the interdiffusion in InGaAs/GaAs material system. ${ }^{15}$ Here, we are more concerned about the effects of a change in strain on the interband transition energy which corresponded to the PL energy in the experiment, other than the strain effects on the In and $\mathrm{Ga}$ (or $\mathrm{Al}$ ) interdiffusion. It is well known that a change in strain changes the band structure and, consequently, the interband transition energy and the PL energy.

Table II compares the interband transition energies as determined by PL measurements for as-grown samples A and $\mathrm{B}$ and calculations that included and excluded strain. The influence of strain on the interband transition energy is significant, being about $66 \mathrm{meV}$ for sample A (QW1) and 96 $\mathrm{meV}$ for sample $\mathrm{B}$ as determined by the calculation for asgrown samples. These values will change after the QWI, as the band edge confinement profiles will no longer be square. Everything else being the same, a reduction in strain should cause a redshift (towards lower photon energy or longer wavelength) or a reduction (suppression) in the blueshift in PL energy in case the QWI is carried out. In this work, the maximum reductions in the blueshift were 16 and $24 \mathrm{meV}$ for samples A (QW1) and B, respectively, as determined from Figs. 4 and 5.

The change in strain in this work was probably due to (1) the interdiffusion, and (2) Ga vacancies injected from the anodic oxide cap, and (3) stress at the interface between the anodic oxide and GaAs resulted from the difference in the thermal expansion coefficients between the anodic oxide and GaAs at RTA temperatures. These three possible causes are discussed below.

For the InGaAs/GaAs QW system, the lattice constant of InGaAs layer is larger than that of the GaAs barrier layer. Compressive strain arises in the well, while tensile stress exists in the GaAs. When In atoms diffuse into the GaAs barrier layer and $\mathrm{Ga}$ atoms diffuse into the well layer, a thin and graded InGaAs interface is formed. After a certain amount of interdiffusion, the in-plane strain is reduced. Thus, the interdiffusion can cause the reduction in the blueshift. However, the interdiffusion also causes a change in the profile of carrier confinement potential, resulting in an increase of the interband transition, and this increase is more than the decrease due to the partial strain relax caused by the interdiffusion. The net effect of interdiffusion is a blueshift, as shown in the experiment by the unanodized samples.

The strain can also be affected by a change in the Ga vacancies due to anodic oxide during the annealing. In the case of the QWI by the $\mathrm{SiO}_{2}$ cap, it is often assumed that $\mathrm{Ga}$ vacancies are produced by the $\mathrm{SiO}_{2}$ cap layer, ${ }^{1}$ and are injected into the semiconductor layers beneath it, thus promoting intermixing. This is mainly due to the relatively high solid solubility of $\mathrm{Ga}$ in $\mathrm{SiO}_{2}$. Ga vacancy injection by the anodic oxide was believed to have taken place in anodic oxide enhanced intermixing in the GaAs/AlGaAs system. ${ }^{13}$

To study how the anodic oxide affects the Ga movements during RTA, we profiled $\mathrm{Al}$ and $\mathrm{Ga}$ from a GaAs/ AlAs/GaAs/substrate reference structure by secondary ion mass spectroscopy (SIMS). The GaAs buffer layer was $\sim 1000 \mathrm{~nm}$ thick. The results were plotted in Fig. 7, showing in-depth profiles of $\mathrm{Al}$ and Ga, taken under identical SIMS conditions. The results were from an anodized, but not annealed, sample and from an annealed sample with half of the surface anodized and half of the surface unanodized before annealing. The Al profiles reveal that during RTA Al atoms were indeed attracted towards the surface by anodic oxide, an indication that anodic oxide enhances the interdiffusion between $\mathrm{Ga}$ and $\mathrm{Al}$ atoms. The $\mathrm{Ga}$ profiles reveal that $\mathrm{Ga}$ concentration in the anodic oxide was increased after RTA, in other words, Ga vacancies were injected by the anodic oxide into the GaAs layer beneath the oxide, and then moved to other layers (including the InGaAs layer). It is known that vacancies induce tensile stress around their sites and will, therefore, be attracted by compressive regions of the material where they can partially relax the imposed stress. 29

For the InGaAs/GaAs and InGaAs/AlGaAs samples studied in this work, when the anodic oxide on the top of the sample injected $\mathrm{Ga}$ vacancies into the materials beneath it, the Ga vacancies reduced the stress and thus the strain in the layers. The Ga vacancies' effects on the interband transition energy are complicated: first, the vacancies reduce the strain and thus lead to a reduction in the blueshift. Second, the 


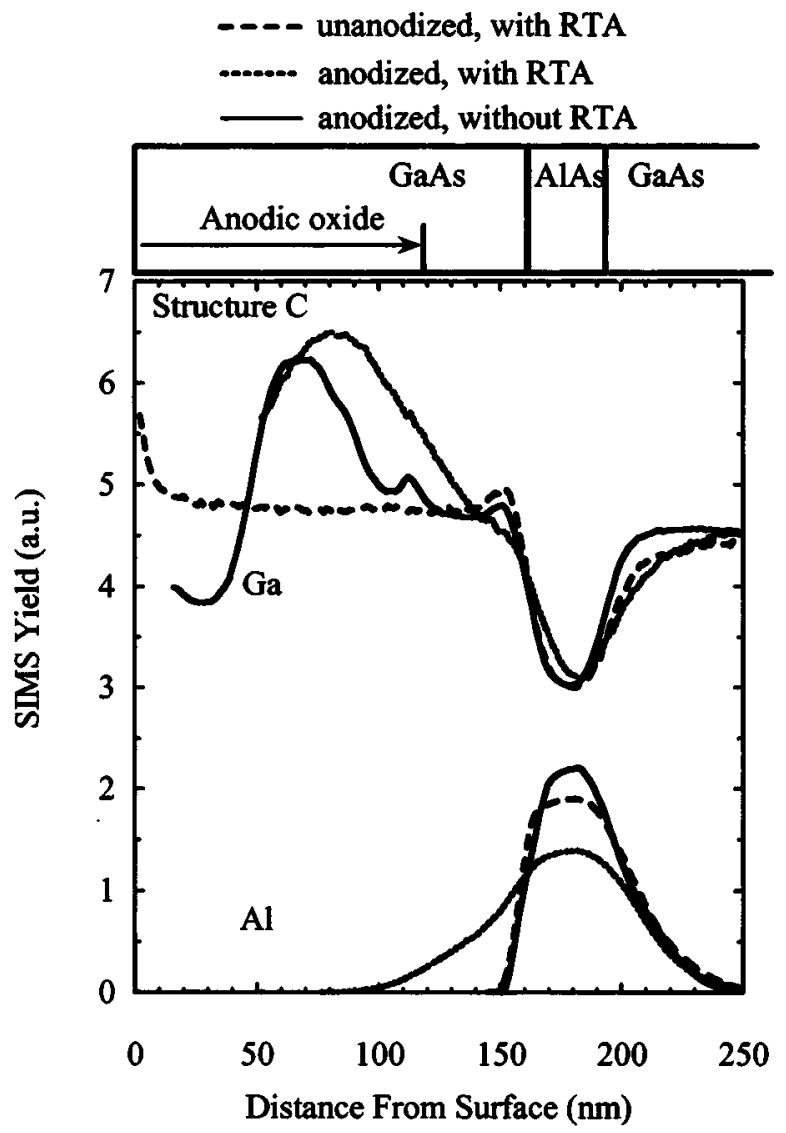

FIG. 7. Secondary ion mass spectroscopy (SIMS) profiles of $\mathrm{Ga}$ and $\mathrm{Al}$ as a function of depth for a GaAs/AlAs/GaAs reference sample. A schematic drawing of the sample structure is shown on the top. The Ga profiles were shifted up for a clear comparison with $\mathrm{Al}$ profiles. Two samples denoted "anodized" were anodized under the same conditions, and a portion of the top GaAs layer was converted into anodic oxide. Two samples were annealed at $900{ }^{\circ} \mathrm{C}$ for $60 \mathrm{~s}$ (denoted as "with RTA").

vacancies enhance the interdiffusion between $\mathrm{Ga}$ and In atoms, which causes the blueshift of the PL energy; The net effect was the reduction in the blueshift in PL energy.

\section{CONCLUSIONS}

Intermixing in InGaAs/AlGaAs quantum well structures was studied with and without an anodic oxide cap by rapid thermal annealing at temperatures in the range from 800 to $930^{\circ} \mathrm{C}$ for durations from 0 to $90 \mathrm{~s}$. The samples were then characterized by low temperature PL measurements at $8 \mathrm{~K}$. Blueshifts in the PL energy were observed in all samples. Anodic oxide has demonstrated to suppress the blueshift noticeably. This is in contrast to the GaAs/AlGaAs system where anodic oxide enhances the blueshift. We believe in both cases anodic oxide enhances the interdiffusion between group III atoms, thanks to the injection of Ga vacancies by the anodic oxide into the layers beneath it. The suppression of the blueshift was attributed to a strain reduction, but the actual mechanism of the strain reduction is not clear yet. The main reason for the strain reduction could be the Ga vacancy injection from the anodic oxide, which reduced the strain, and at the mean time also enhances interdiffusion between In and $\mathrm{Ga}$ or $\mathrm{Al}$ atoms.

\section{ACKNOWLEDGMENTS}

The authors are grateful to Dr. M. Petravic for the SIMS measurements and Dr. S. F. Yu for careful reading of the manuscript. The authors appreciate helpful discussions with Professor C. Jagadish and Professor M. Gal and Dr. L. Fu.

${ }^{1}$ Quantum Well Intermixing for Photonics, edited by E. H. Li (SPIE Milestone Series, Bellingham 1997).

${ }^{2}$ E. H. Li, B. L. Weiss, and K. S. Chan, Phys. Rev. B 46, 15181 (1992).

${ }^{3}$ S. Yuan, N. Frank, G. Bauer, and M. Kriechbaum, Phys. Rev. B 50, 5286 (1994).

${ }^{4}$ H. H. Tan, J. S. Williams, C. Jagadish, P. T. Burke, and M. Gal, Appl. Phys. Lett. 68, 2401 (1996).

${ }^{5}$ D. G. Deppe and H. Holonyak, Jr., J. Appl. Phys. 64, R93 (1988)

${ }^{6}$ J. H. Marsh, Semicond. Sci. Technol. 8, 1136 (1993).

${ }^{7}$ S. Burkner, M. Maier, E. C. Larkins, W. Rothemound, E. P. O'Reilly, and J. D. Ralston, J. Electron. Mater. 24, 805 (1995).

${ }^{8}$ R. M. Cohen, Mater. Sci. Eng., R. 20 (1997).

${ }^{9}$ A. McKee, C. J. McLean, G. Lullo, A. C. Bryce, R. M. De La Rue, J. H. Marsh, and C. C. Button, IEEE J. Quantum Electron. 33, 45 (1997).

${ }^{10}$ S. Yuan, Y. Kim, C. Jagadish, P. T. Burke, M. Gal, J. Zou, D. Q. Cai, D. J. H. Cockayne, and R. M. Cohen, Appl. Phys. Lett. 70, 1269 (1997).

${ }^{11}$ S. Yuan, Y. Kim, H. H. Tan, C. Jagadish, P. T. Burke, L. V. Dao, M. Gal, J. Zou, D. Q. Cai, D. J. H. Cockayne, and R. M. Cohen, J. Appl. Phys. 83, 1305 (1998).

${ }^{12}$ Y. Kim, S. Yuan, R. Leon, C. Jagadish, M. Gal, M. Johnston, M. Phillips, M. Stevens Kalceff, J. Zou, and D. Cockayne, J. Appl. Phys. 80, 5014 (1996).

${ }^{13}$ S. Yuan, C. Jagadish, Yong Kim, Y. Chang, H. H. Tan, R. M. Cohen, M. Petravic, L. V. Dao, M. Gal, M. C. Y. Chan, E. H. Li, and P. S. Zory, IEEE J. Sel. Top. Quantum Electron. 4, 629 (1998).

${ }^{14}$ S. W. Lee, E. H. Li, and G. Karunasiri, Appl. Phys. Lett. 74, 1102 (1999).

${ }^{15}$ W. P. Gillin, J. Appl. Phys. 85, 790 (1999).

${ }^{16}$ S.-W. Ryu, I. Kim, B.-D. Choe, and W. G. Jeong, Appl. Phys. Lett. 67, 1417 (1995)

${ }^{17}$ G. Li, S. J. Chua, and S. J. Xu, X. C. Wang, L. Ke, and J. H. Marsh, Appl. Phys. Lett. 73, 3393 (1998)

${ }^{18}$ O. M. Khreis, W. P. Gillin, and K. P. Homewood, Phys. Rev. B 55, 15813 (1997).

${ }^{19}$ H. H. Tan, S. Yuan, M. Gal, and C. Jagadish, in Semiconductor Quantum Well Intermixing, edited by E. Herbert Li (Gordon and Breach, Amsterdam, 2000), p. 307.

${ }^{20}$ M. J. Grove, D. A. Hudson, P. S. Zory, R. J. Dalby, C. M. Harding, and A. Rosenberg, J. Appl. Phys. 76, 587 (1994).

${ }^{21}$ It should be noted here that the definition of $L_{d}$ varies in the literature; for instance, some authors define $L_{d}$ as $2 \sqrt{D t}$, see K. Kash et al., J. Appl. Phys. 63, 190 (1988), but the factor of 2 is present in Eq. (1).

${ }^{22}$ J. Crank, The Mathematics of Diffusion, 2nd ed. (Oxford University, Oxford, U.K., 1975).

${ }^{23}$ M. Abramowitz and I. A. Stegun, Handbook of Mathematical Functions Washington, D. C. (National Bureau of Standards, Washington, DC, 1964).

${ }^{24}$ D. Ahn and S. L. Chuang, IEEE J. Quantum Electron. 24, 2400 (1988).

${ }^{25}$ S. L. Chuang, Phys. Rev. B 43, 9649 (1991).

${ }^{26}$ D. J. BenDaniel and C. B. Duke, Phys. Rev. 152, 683 (1966).

${ }^{27}$ For the mechanism of thermal induced partial strain relieving, see, e.g., K. G. Budinski and M. K. Budinski, Engineering Materials Properties and Selection, 7th ed. (Person, NJ, 2002), p. 367.

${ }^{28}$ P. Cusumano, B. S. Ooi, A. S. Helmy, S. G. Ayling, A. C. Bryce, J. H. Marsh, B. Voegele, and M. J. Rose, J. Appl. Phys. 81, 2445 (1997).

${ }^{29}$ A. Pepin, C. Vieu, M. Schneider, H. Launois, and Y. Nissim, J. Vac. Sci. Technol. B 15, 142 (1997). 